ORIGINAL

\title{
Estudio poblacional sobre el estado de salud y nutrición de habitantes de la ciudad de Mérida, México
}

\author{
Enrique Efraín Barbosa-Martín ${ }^{a}$,* Ileana Fajardo-Niquete ${ }^{a}$, Fernando Sosa-Valadez ${ }^{a}$, \\ Francisco Cetina-Sánchez ${ }^{a}$, Ivan Puc-Encalada ${ }^{a}$, Rafael Vargas-Espinosa ${ }^{a}$, \\ Rebeca Jiménez-Estrada ${ }^{a}$, David Abram Betancur-Ancona
}

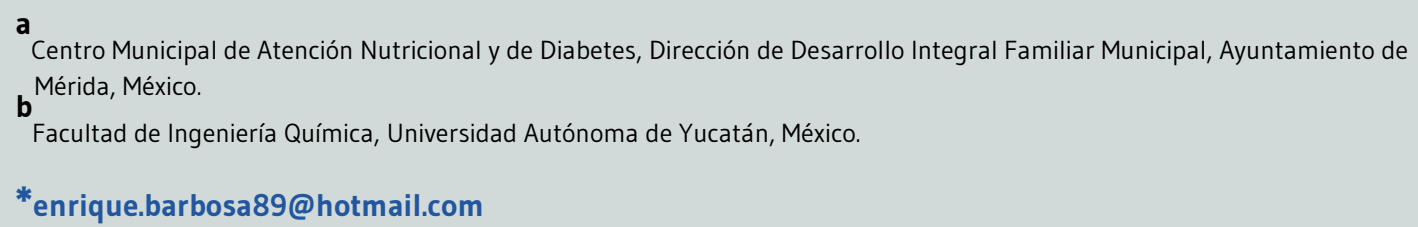

Recibido el 25 de febrero de 2016; aceptado el 20 de junio de 2016.

Estudio poblacional sobre el estado de salud y nutrición de habitantes de la ciudad de Mérida, México

PALABRAS CLAVE
Hipertensión Arterial;
Diabetes Mellitus;
Desnutrición;
Sobrepeso;
Obesidad;
México.

\section{RESUMEN}

Introducción: La Encuesta Nacional de Salud y Nutrición (ENSANUT) reporta el panorama actual de la epidemiología nutricional nacional y estatal en México. Sin embargo, no existen reportes nutricionales de las capitales de los estados mexicanos. El presente trabajo tuvo como objetivo evaluar el estado de salud y nutrición en habitantes de Mérida, Yucatán, México.

Material y Métodos: Estudio transversal, retrospectivo y descriptivo que utilizó los lineamientos metodológicos y diagnósticos de la ENSANUT, OMS y Normas Oficiales Mexicanas. Se determinaron casos probables de hipertensión arterial sistémica (CPHA), casos probables de diabetes mellitus (CPDM) y se realizaron mediciones antropométricas para establecer prevalencias de desnutrición, normopeso y exceso de peso. Se utilizó SPSS 20.0 y Excel 2013 para calcular proporciones y expresar los resultados en prevalencias.

Resultados: Se evaluaron 4.324 sujetos (644 preescolares, 1.881 escolares, 133 adolescentes y 1.666 adultos). En adultos, la prevalencia de CPHA fue $11,8 \%$ y la de CPDM 10,2\%. En preescolares las prevalencias de bajo peso, emaciación y talla baja fueron $0,4 \%, 0,7 \%$ y 2,7\%, respectivamente. En escolares, adolescentes y adultos las prevalencias de desnutrición fueron 3,3\%, 2,2\% y $1,2 \%$, respectivamente. Las proporciones de normopeso y de exceso de peso en preescolares, escolares, adolescentes y adultos fueron $54,6 \%, 45,5 \%, 48,1 \%$ y $20,7 \%$ y del $16,1 \%, 51 \%, 49,5 \%$ y $77,9 \%$, respectivamente.

Conclusiones: Se evidenciaron altas prevalencias de casos probables de diabetes mellitus en hombres y bajas prevalencias de desnutrición en todas las edades. La proporción de exceso de peso en menores de 20 años fue mayor a lo reportado tanto a nivel nacional como estatal. 


\section{KEYWORDS}

Hypertension;

Diabetes Mellitus;

Malnutrition;

Overweight;

Obesity;

\section{Health and nutritional status in Mérida, México: A population-based study}

\section{ABSTRACT}

Introduction: The Health and Nutrition National Survey (ENSANUT) reports the panorama of the national and state nutritional epidemiology in Mexico. However, there are no reports of the capital cities of the country. The objective of this report was to evaluate the health and nutritional condition in a sample of the population of Merida, Yucatan, Mexico.

Material and Methods: Transversal, retrospective and descriptive study that used the methodological and diagnostic guidelines of ENSANUT, WHO and Official Mexican Standards. To know malnutrition, normal weight and overweight prevalence, hypertension probable cases (CPHA) and diabetes mellitus probable cases (CPDM) were determined and anthropometric measurements were performed. SPSS 20.0 and Excel 2013 were used to calculate proportions and the results were expressed in prevalence.

Results: 4,324 subjects were evaluated (644 pre-school pupils, 1,881 school pupils, 133 adolescents and 1,666 adults). In adults the prevalence of CPHA and CPDM were $11.8 \%$ and $10.2 \%$, respectively. For pre-school pupils the prevalence of low weight, emaciation and low height were $0.4 \%, 0.7 \%$ and $2.7 \%$, respectively. For school pupils, adolescents and adults malnutrition prevalence was $3.3 \%, 2.2 \%$ and $1.2 \%$ respectively. The prevalence of normal weight and overweight in pre-school pupils, school pupils, adolescents and adults was $54.6 \%, 45.5 \%, 48.1 \%, 20.7 \%$ and $16.1 \%, 51 \%, 49.5 \%$ y $77.9 \%$, respectively.

Conclusions: High prevalence of diabetes mellitus probable cases in men and low prevalence of malnutrition in all ages were observed. The prevalence of overweight in pre-school pupils, school pupils and adolescents was higher than the national and estate average.

\section{CITA}

Barbosa-Martín EE, Fajardo-Niquete I, Sosa-Valadez F, Cetina-Sánchez F, Puc-Encalada I, Vargas-Espinosa R, Jiménez-Estrada R, Betancur-Ancona DA. Estudio poblacional sobre el estado de salud y nutrición de habitantes de la ciudad de Mérida, México. Rev Esp Nutr Hum Diet. 2016; 20(3): 208 - 215. doi: 10.14306/renhyd.20.3.217

\section{INTRODUCCIÓN}

Los esfuerzos de las instituciones de salud en México en materia de epidemiología nutricional se han enfocado principalmente al diagnóstico de salud y nutrición, a través del Sistema Nacional de Encuestas de Salud, que desde los años ochenta ha aplicado diversas herramientas de recolección de datos en la población mexicana. La Encuesta Nacional de Salud y Nutrición (ENSANUT), observación más reciente de dicha estrategia, permitió desde el año 2006 contar con información actualizada y detallada sobre las características de los hogares, los gastos familiares en alimentación, diversas prácticas de salud así como las prevalencias de desnutrición y exceso de peso, entre otras cuestiones, a partir de muestras representativas tanto del país como de las entidades federativas. No obstante, pese a que actualmente se cuenta con estos datos nacionales y estatales, aún no hay reportes nutricionales oficiales de las capitales de los estados mexicanos ${ }^{1,2}$.

La ENSANUT ha demostrado que en la población convergen alarmantemente dos condiciones crónicas opuestas, productos de la mala alimentación de los mexicanos: la desnutrición, que afecta principalmente a niños, y el exceso de peso, que se ha agravado en la población adulta.

Por un lado, la desnutrición infantil se inicia durante la gestación y los primeros dos años de vida y tiene efectos adversos sobre la salud de los niños ${ }^{3}$. Se estima que 178 millones de niños menores de cinco años en el mundo sufren de desnutrición crónica (baja talla para la edad), la cual es responsable de $35 \%$ de muertes en este grupo de edad 4 . En México, $2,8 \%, 13,6 \%$ y $1,6 \%$ de los niños en edad preescolar padecen bajo peso, talla baja y emaciación, respectivamente ${ }^{1}$. En el estado de Yucatán, la prevalencia de talla baja rebasa la media nacional². 
Por otro lado, la ENSANUT muestra que el exceso de peso está presente en 3 de cada 10 niños y adolescentes y en 7 de cada 10 adultos mexicanos ${ }^{3}$. En Yucatán, la prevalencia en niños y adolescentes rebasa en más de diez puntos porcentuales lo reportado a nivel nacional ${ }^{4}$. Esta condición se convierte en alarmante, al tener en cuenta tanto las comorbilidades que conlleva como hipertensión, diabetes y dislipidemia ${ }^{5}$, así como la fuerte carga financiera que genera para el sistema de salud pública ${ }^{6}$.

Es por lo anterior que en el presente trabajo de investigación se tuvo como objetivo evaluar el estado de salud y de nutrición en una muestra de habitantes del municipio de Mérida, capital del estado de Yucatán, mediante la valoración de presión arterial, de glicemia capilar y de indicadores antropométricos.

\section{MATERIAL Y MÉTODOS}

Estudio transversal, retrospectivo y descriptivo realizado durante septiembre de 2012 y agosto de 2015. Los sujetos de estudio fueron los pertenecientes al padrón de beneficiarios de dos programas de la Dirección de Desarrollo Integral Familiar Municipal (DIF) de los Ayuntamientos de la Ciudad de Mérida entre 2012-2015 y 2015-2018: Ferias de Salud Públicas y Visitas Escolares. Dichos eventos se llevaron a cabo en 121 centros públicos y privados distribuidos tanto en la ciudad $(22,31 \%$ en el centro, $19,83 \%$ en el sur, $18,18 \%$ en el norte, $17,36 \%$ en el este y $11,57 \%$ en el oeste) como en comisarías de Mérida (10,74\%). Todos los procedimientos utilizados siguieron las normas éticas de la Declaración de Helsinki y la participación de los sujetos en el estudio fue previo consentimiento informado.

Se realizó aplicando los lineamientos metodológicos dictados tanto en la ENSANUT Nacional ${ }^{3}$, como en la ENSANUT Yucatán ${ }^{4}$, realizando las siguientes modificaciones: en la valoración del estado de salud, este estudio abarcó la determinación de Casos Probables de Hipertensión Arterial Sistémica (CPHA) y de Casos Probables de Diabetes Mellitus (CPDM) en adultos mediante la toma de muestras; asimismo, en la evaluación nutricional, se incluyó la identificación de desnutrición, normopeso y exceso de peso en todos los grupos de edad.

Evaluación del estado de salud: Para la valoración de la presión arterial se usaron tensiómetros aneroides Hergom ${ }^{\circledR}$ y los resultados se compararon con lo referenciado por la Norma Oficial Mexicana para la prevención, detección, diagnóstico, tratamiento y control de la hipertensión arterial sistémica", la cual identifica como "Caso Probable de
Hipertensión Arterial Sistémica" a la persona que en una toma ocasional obtenga una cifra de presión sistólica $>140 \mathrm{mmHg}$ y/o presión diastólica $>90 \mathrm{mmHg}$. Se consideraron como datos válidos los valores de presión arterial sistólica entre 80 y $200 \mathrm{mmHg}$ y de presión arterial diastólica entre 50 y $110 \mathrm{mmHg}^{3}$. La glicemia alterada se determinó mediante pruebas de punción capilar, utilizando glucómetros Optium Xceed Abbott ${ }^{\circledR}$ y tiras de prueba FreeStyle Optium Abbot ${ }^{\circledR}$. El diagnóstico se estableció en función a los lineamientos de la Norma Oficial Mexicana para la prevención, tratamiento y control de la diabetes mellitus ${ }^{8}$, la cual categoriza como "Caso Probable de Diabetes Mellitus" a la persona que presente una glicemia capilar casual $>140 \mathrm{mg} / \mathrm{dL}$.

Evaluación del estado de nutrición: Se obtuvieron mediciones de longitud y peso, en niños menores de cinco años, así como talla y peso, en sujetos mayores de cinco años. Los datos obtenidos se compararon con los Patrones de Crecimiento Infanti $\left.\right|^{9,10}$ y con los Criterios Diagnósticos por Índice de Masa Corporal (IMC) para adultos ${ }^{11}$, establecidos por la Organización Mundial de la Salud (OMS). La valoración de desnutrición se hizo en niños menores de cinco años, utilizando los tres indicadores antropométricos estándar: peso para la edad, longitud/talla para la edad y peso para la longitud/talla. Los diagnósticos de desnutrición para cada uno de ellos (bajo peso, talla baja y emaciación, respectivamente) se aplicaron en aquellos niños cuyo puntaje $Z$ fue $<-2$. La determinación de normopeso y de exceso de peso se realizó utilizando el indicador IMC/edad en preescolares, escolares y adolescentes y el IMC en adultos. En los preescolares y escolares se tomaron en cuenta únicamente los valores de IMC entre 10 y 38, y en adolescentes y adultos entre 10 y 58 . Asimismo, solamente se contemplaron sujetos adultos con tallas entre 1,30 y $2,0 \mathrm{~m}$ y con ausencia de embarazo $0^{3,4}$.

Análisis de los datos: Se utilizaron los programas Statistical Package for the Social Sciences (SPSS) versión 20.0 y Microsoft Excel 2013, calculando proporciones y expresando los resultados en prevalencias. Este trabajo expresa parámetros de estudio al incluir a todo el universo de sujetos adscritos al padrón de beneficiarios de los dos programas del DIF Mérida.

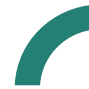

\section{RESULTADOS}

El universo de sujetos evaluados fue de 4.324, categorizados en cuatro grupos de edad: 644 preescolares (337 niños y 307 niñas), 1.881 escolares (964 niños y 917 niñas), 133 adolescentes (53 hombres y 80 mujeres) y 1.666 adultos (285 hombres y 1.381 mujeres). Del total de adultos (1.666), 
la evaluación de la presión arterial y de la glucosa capilar se realizó en 1.107 y 1.021 sujetos, respectivamente. Los rangos de edad contemplados fueron de 0-4 años, 5-11 años, 12-19 años y $\geq 20$ años para los preescolares, escolares, adolescentes y adultos, respectivamente ${ }^{3,4}$.

\section{Estado de salud}

Casos probables de Hipertensión Arterial Sistémica (CPHA) y de Diabetes Mellitus (CPDM): De los 1.107 adultos evaluados, 233 fueron hombres y 946 mujeres. Los resultados indicaron que la prevalencia de CPHA fue del $11,8 \%$. El análisis por sexo reveló que en los hombres, la media municipal fue del $22,7 \%$ y en las mujeres del 9,19\% (Figura 1). En la valoración de la glicemia capilar se diagnosticaron 1.021 adultos; 235 hombres y 786 mujeres. La proporción municipal encontrada de CPDM fue del 10,2\% para la población en general, del 13,1\% para los hombres y 9,4\% para las mujeres (Figura 2).

Figura 1. Prevalencia de hipertensión arterial nacional ${ }^{3}$ y estatal ${ }^{4}$ y de CPHA municipal en adultos mayores de 20 años.

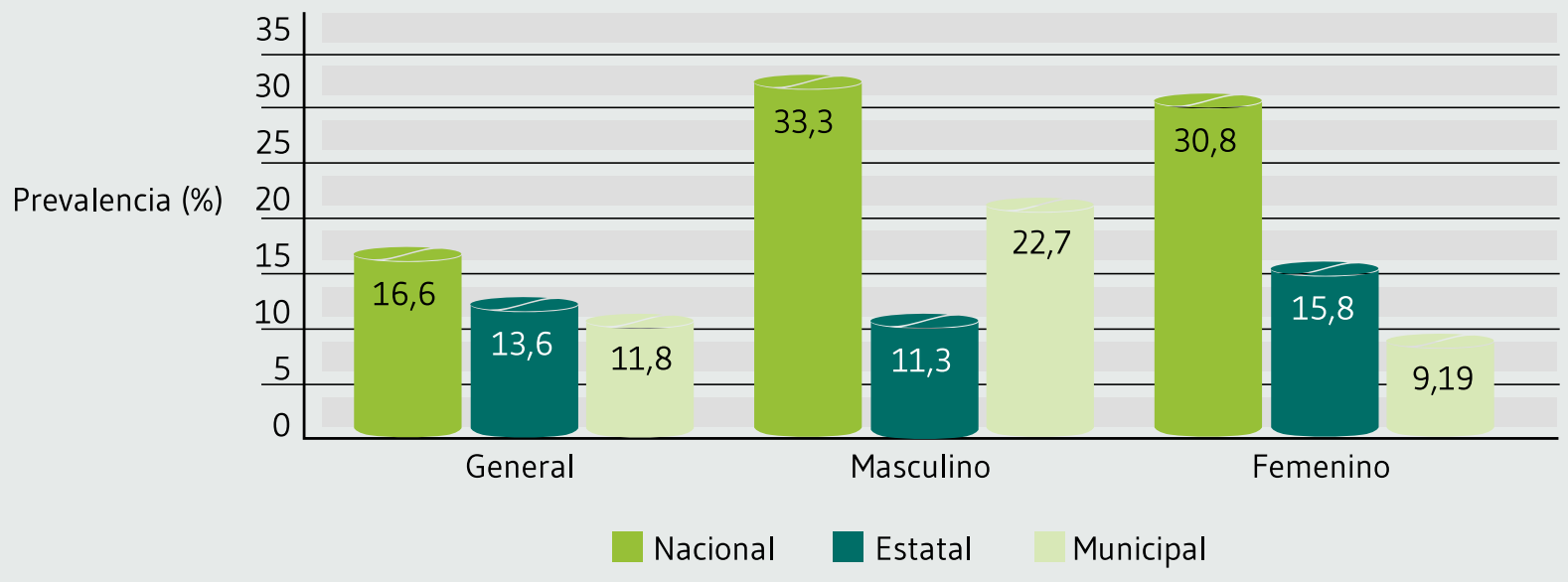

Figura 2. Prevalencia de diabetes mellitus nacional ${ }^{3}$ y estatal ${ }^{4}$ y de CPDM municipal de en adultos mayores de 20 años

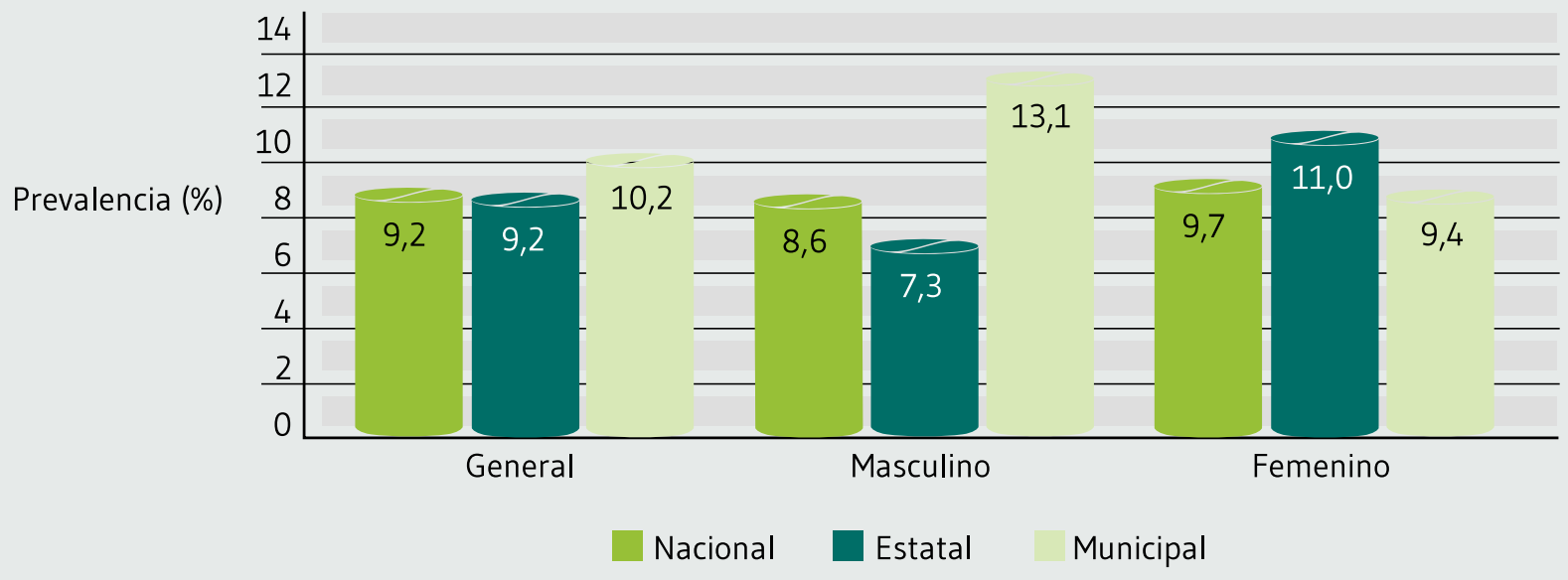




\section{Estado de nutrición}

Desnutrición: Los datos analizados para la población preescolar indicaron que la prevalencia de bajo peso fue del $0,4 \%$, la de emaciación del 0,7\% y la de talla baja del 2,7\% (Figura 3). La valoración por sexo reveló que la presencia de bajo peso fue tres veces menor en niños $(0,2 \%)$ que en niñas $(0,6 \%)$. En tanto a emaciación, la proporción fue cuatro veces mayor en niños $(1,1 \%)$ que en niñas $(0,3 \%)$ y en talla baja se evidenció una diferencia de 1,6 puntos porcentuales (3,5\% en niños y 1,9\% en niñas). En escolares, adolescentes y adultos las prevalencias generales de desnutrición fueron del $3,3 \%$ ( $4,2 \%$ en niños y $2,3 \%$ en niñas), $2,2 \%$ ( $5,6 \%$ en hombres y $0 \%$ en mujeres) y $1,2 \%$ ( $1,7 \%$ en hombres y $1,0 \%$ en mujeres), respectivamente.
Normopeso y exceso de peso: Las prevalencias generales de normopeso en los preescolares, escolares, adolescentes y adultos fueron del $54,6 \%$ (52,2\% en niños y $57,3 \%$ en niñas), $45,5 \%$ ( $41,8 \%$ en niños y $49,5 \%$ en niñas), $48,1 \%$ ( $43,3 \%$ en hombres y $51,2 \%$ en mujeres) y $20,7 \%$ ( $16,1 \%$ en hombres y $21,7 \%$ en mujeres), respectivamente. En tanto a la identificación de exceso de peso (sobrepeso más obesidad), la proporción encontrada en la población preescolar fue del $16,1 \%$ y en la población escolar del $51 \%$. En los adolescentes y adultos las proporciones fueron del $49,5 \%$ y $77,9 \%$, respectivamente (Tabla 1). En el análisis por sexo se observó que la prevalencia de exceso de peso en preescolares fue mayor en los niños (18,9\%) que en las niñas (13,0\%). Esta condición se replicó en los escolares y adolescentes masculinos donde se

Figura 3. Prevalencia nacional ${ }^{3}$, estatal ${ }^{4}$ y municipal de desnutrición en niños menores de 5 años.

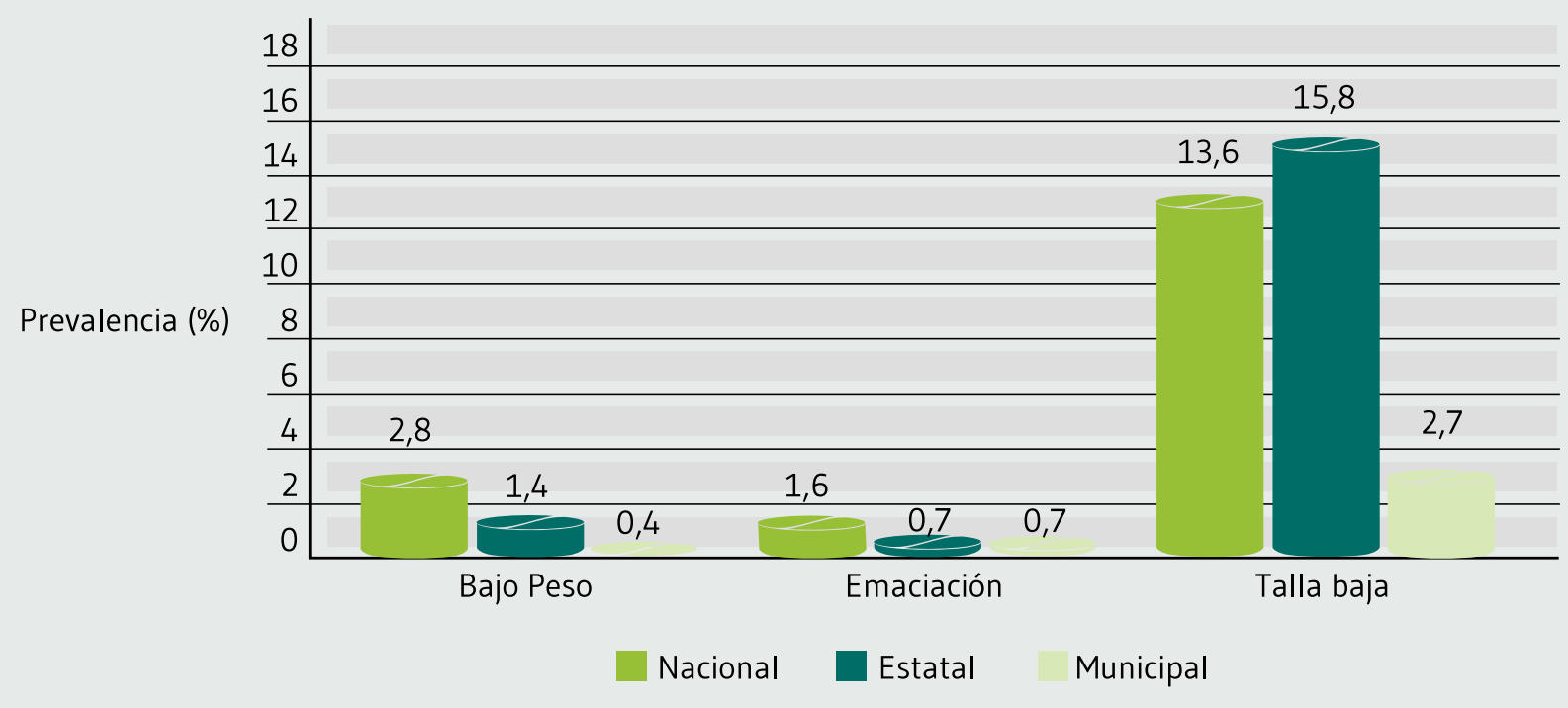

Tabla 1. Prevalencias nacional ${ }^{3}$, estatal ${ }^{4}$ y municipal de normopeso y exceso de peso por grupo de edad.

\begin{tabular}{|c|c|c|c|c|c|c|c|c|c|c|c|c|}
\hline \multirow{2}{*}{ Condición } & \multicolumn{3}{|c|}{ Preescolares } & \multicolumn{3}{|c|}{ Escolares } & \multicolumn{3}{|c|}{ Adolescentes } & \multicolumn{3}{|c|}{ Adultos } \\
\hline & $\mathbf{N}$ & E & M & N & E & $M$ & N & E & M & $\mathbf{N}$ & E & $M$ \\
\hline Normopeso & NR & NR & 54,6 & NR & NR & 45,5 & NR & NR & 48,1 & NR & NR & 20,7 \\
\hline Exceso de peso & 9,7 & 14,6 & 16,1 & 34,4 & 45,1 & 51,0 & 34,9 & 43,4 & 49,5 & 71,2 & 80,3 & 77,9 \\
\hline Sobrepe & & & & 19,8 & 22,4 & 25,5 & 21,6 & 28,0 & 25,5 & 38,8 & 35,5 & 38,8 \\
\hline Obe & & & & 14,6 & 22,7 & 25,5 & 13,3 & 15,4 & 24,0 & 32,4 & 44,8 & 39,1 \\
\hline
\end{tabular}

E: Estatal; M: Municipal; N: Nacional; NR: Datos no reportados. 
observaron altas prevalencias de obesidad, $28,8 \%$ y $37,7 \%$ respectivamente. En el caso de los adultos las proporciones de sobrepeso y de obesidad fueron próximas al $40 \%$ en ambos sexos (Tabla 2).

\section{Discusión}

Al comparar la proporción de CPHA encontrada en el presente trabajo $(11,8 \%)$, con las prevalencias nacional $(16,6 \%)^{3}$ y estatal $(13,6 \%)^{4}$ de hipertensión arterial por diagnóstico médico previo, se observa que en la muestra municipal existe una menor proporción de esta condición (Figura 1). El análisis por sexo revela que para los hombres, la prevalencia de la muestra municipal $(22,7 \%)$ es menor que la nacional $(33,3 \%)^{3}$, pero mayor que la estatal $(11,3 \%)^{4}$. En las mujeres, la media municipal encontrada $(9,19 \%)$ es casi un tercio y casi la mitad de lo reportado a nivel nacional $(30,8 \%)^{3}$ y estatal $(15,8 \%)^{4}$, respectivamente. Los resultados municipales concuerdan con lo reportado por Arroyo y Cols. ${ }^{12}$, quienes, en un estudio con adultos jóvenes de la ciudad de Mérida, encontraron menores prevalencias de hipertensión en mujeres $(9,4 \%)$ que en hombres $(15,5 \%)$. Los autores concluyen que esta condición podría estar asociada a diversos factores de riesgo, tales como la elevada prevalencia de obesidad central, el sobrepeso elevado, el consumo de una dieta de alta densidad energética y patrones sedentarios de actividad física.

La proporción de CPDM (10,2\%) es 1,0\% mayor a lo reportado para diabetes mellitus por diagnóstico médico previo, tanto a nivel nacional $(9,2 \%)^{3}$ como estatal $(9,2 \%)^{4}$. Destaca que, en el caso de los hombres, la prevalencia encontrada $(13,1 \%)$ fue 4,5 puntos porcentuales arriba de la media nacional $(8,6 \%)^{3}$ y 5,8 arriba de la media estatal $(7,3 \%)^{4}$. En las mujeres, la prevalencia de la muestra municipal (9,4\%) fue $1,6 \%$ por debajo de la media estatal $(11 \%)^{4}$ y muy similar a la media nacional $(9,7 \%)^{3}$ (Figura 2$)$. En este sentido, desde los años sesenta existen datos sobre la prevalencia de diabetes mellitus en diversas muestras de la población de Mérida. Para 1968, 1989 y 2011 las prevalencias reportadas fueron del $3,7 \%^{13}, 12 \%^{13}$ y $12,7 \%^{14}$, respectivamente. La razón de estas prevalencias se fundamenta, por una parte, en la presencia de factores de riesgo de la enfermedad como exceso de peso e hipertensión arterial ${ }^{15}$. Por otro lado, la epidemiología también parece responder a una posible influencia genética. Esto, según lo reportado por Rosado y Cols. ${ }^{16}$, debido a la predisposición genética por la ascendencia maya de los habitantes de esta región.

En relación al diagnóstico de desnutrición en niños menores de cinco años, se observa que en la muestra municipal existen bajas prevalencias en todos los indicadores valorados. Destaca que la prevalencia municipal de bajo peso $(0,4 \%)$ es siete veces menor a la nacional $(2,8 \%)^{3}$ y 3,5 veces menor a la estatal $(1,4 \%)^{4}$. En tanto a la prevalencia de emaciación $(0,7 \%)$, esta es casi la mitad de la nacional $(1,6 \%)^{3}$ e igual que la estatal $(0,7 \%)^{4}$. La discrepancia más notoria se observa en la talla baja municipal $(2,7 \%)$, donde la diferencia con las medias nacional $(13,6 \%)^{3}$ y estatal $(15,8 \%)^{4}$ es de más de diez puntos porcentuales (Figura 3). Diversos autores concuerdan con que esta situación responde a la asociación entre el exceso de peso y los períodos de crecimiento acelerado (como la etapa preescolar y la escolar), ya que el aumento de peso precipitado durante los primeros años de vida se asocia con el crecimiento lineal exacerbado $17,18,19$ Este argumento cobra relevancia al considerar el exceso de peso observado en los preescolares y escolares de este estudio (Tabla 2). Por otro lado, las proporciones de desnutrición encontradas en los escolares y adolescentes fueron relativamente bajas $(3,3 \%$ y $2,2 \%$, respectivamente) y estas

Tabla 2. Prevalencias municipales de normopeso y exceso de peso por sexo y por grupo de edad.

\begin{tabular}{|c|c|c|c|c|c|c|c|c|c|c|c|c|}
\hline \multirow[t]{2}{*}{ Condición } & \multicolumn{3}{|c|}{ Preescolares } & \multicolumn{3}{|c|}{ Escolares } & \multicolumn{3}{|c|}{ Adolescentes } & \multicolumn{3}{|c|}{ Adultos } \\
\hline & $\mathrm{H}$ & M & G & $\mathrm{H}$ & M & G & $\mathrm{H}$ & M & G & H & M & G \\
\hline Normopeso & 52,2 & 57,3 & 54,6 & 41,8 & 49,5 & 45,5 & 43,3 & 51,2 & 48,1 & 16,1 & 21,7 & 20,7 \\
\hline Exceso de peso & 18,9 & 13,0 & 16,1 & 53,9 & 48,0 & 51,0 & 50,9 & 48,7 & 49,5 & 82,0 & 77,1 & 77,9 \\
\hline Sobrepeso & \multirow{2}{*}{18,9} & \multirow{2}{*}{13,0} & \multirow{2}{*}{16,1} & 25,1 & 26,0 & 25,5 & 13,2 & 33,7 & 25,5 & 41,7 & 38,2 & 38,8 \\
\hline Obesidad & & & & 28,8 & 22,0 & 25,5 & 37,7 & 15,0 & 24,0 & 40,3 & 38,9 & 39,1 \\
\hline
\end{tabular}


concuerdan con los resultados de Alcocer-Aguilar y Cols. ${ }^{20}$, quienes hallaron desnutrición en el $6,4 \%$ de una muestra de 233 habitantes de Mérida, de entre 9 y 14 años.

La proporción de exceso de peso (sobrepeso más obesidad) encontrada en todos los sujetos menores de 20 años resultó ser mayor a lo reportado tanto a nivel nacional como estatal. En el caso de los preescolares, la prevalencia de la muestra municipal $(16,1 \%)$ fue casi el doble de la media nacional $(9,7 \%)^{3}$ y $1,5 \%$ mayor que la media estatal $(14,6 \%)^{4}$ (Tabla 1). En un estudio observacional realizado por Torres y Cols. ${ }^{21}$, donde se evaluaron indicadores dietéticos y antropométricos en 91 sujetos menores de dos años residentes de Mérida, se observa una prevalencia de exceso de peso similar a la del presente estudio (16,5\%). Según los autores, esta prevalencia parece estar relacionada con prácticas inadecuadas de alimentación infantil; tales como el inicio precoz de la alimentación complementaria, el exceso de calorías ingeridas y que solamente la mitad de la población estudiada poseía antecedentes de lactancia materna exclusiva. En los escolares y adolescentes las prevalencias municipales (51\% y $49,5 \%$, respectivamente) también fueron mayores que las nacionales $(34,4 \% \text { y } 34,9 \% \text {, respectivamente })^{3}$ y que las estatales (45,1\% y 43,4\%, respectivamente) ${ }^{4}$ (Tabla 1$)$. En este sentido, el trabajo de Alcocer-Aguilar y Cols. ${ }^{20}$, Ilevado a cabo con 233 meridanos de entre 9 y 14 años, encontró una proporción de sujetos con exceso de peso del 40,3\%. En ambos estudios municipales la prevalencia es alarmantemente elevada y la razón de ello puede encontrar sustento en el trabajo cualitativo de Méndez y Cols. ${ }^{22}$. Estos investigadores analizaron las percepciones de 48 cuidadores de sujetos obesos meridanos de entre 6 y 13 años y, con base en eso, evidenciaron un alto consumo de productos de harina refinada (galletas y pastelillos), la sustitución de agua por bebidas azucaradas, así como diversas prácticas sedentarias. De igual manera, los autores reportan que más de la mitad de los cuidadores no reconocieron a la obesidad como un verdadero riesgo para la salud de los infantes. En la población adulta, la proporción municipal de exceso de peso $(77,9 \%)$ resultó ser mayor que la nacional $(71,2 \%)^{3}$, pero menor que la estatal $(80,3 \%)^{4}$ (Tabla 1$)$. Estos resultados municipales coinciden con lo reportado por Arroyo y Cols. ${ }^{12}$, quienes haIlaron prevalencias que oscilaron entre el $55 \%$ y el $90 \%$ en diversos grupos rurales y urbanos del estado de Yucatán.

El problema del exceso de peso ha sido perfectamente caracterizado y su origen, cuando no está asociado a patología alguna, se encuentra en el mayor consumo de energía aunado a un estilo de vida sedentario y a una predisposición genéti$\mathrm{ca}^{23}$. Gran parte del riesgo que conlleva esta condición se debe a que está asociada al desarrollo de diversas enfermedades, tales como diabetes mellitus tipo 2, dislipidemia, cáncer, enfermedad cardiovascular y apnea obstructiva del sueño, entre otras $^{24}$. De igual manera, el sobrepeso y la obesidad son problemas progresivos, ya que el riesgo de padecerlos incrementa conforme el individuo es de mayor edad ${ }^{25}$. Esto justifica los resultados del presente estudio en donde observamos un incremento gradual en las prevalencias municipales de exceso de peso: $16,1 \%$ en preescolares, $51 \%$ en escolares, $49,5 \%$ en adolescentes y $77,9 \%$ en adultos (Tabla 1 ).

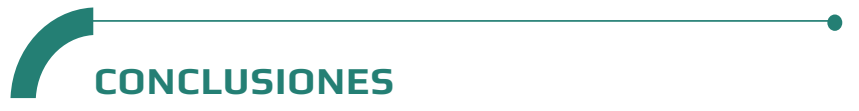

En comparación con los resultados nacionales y estatales, en la muestra municipal de adultos existen bajas prevalencias de CPHA; sólo en los hombres se rebasa la media estatal. La prevalencia de CPDM es menor únicamente en mujeres, tanto a nivel nacional como estatal. En los cuatro grupos de edad estudiados se observan bajas prevalencias de todos los indicadores de desnutrición. Contrariamente, las proporciones de exceso de peso en los sujetos menores de 20 años son mayores a lo reportando tanto a nivel nacional como estatal. En los adultos, la proporción municipal de exceso de peso es mayor que la nacional pero menor que la estatal. Los hallazgos de este trabajo serán de gran relevancia para dirigir y plantear estrategias de promoción a la salud desde todos los ejes de intervención, debido a que los resultados obtenidos en la mayoría de los parámetros muestran tendencias que difieren con el escenario tanto nacional como estatal, e incluso con algunos reportes municipales.

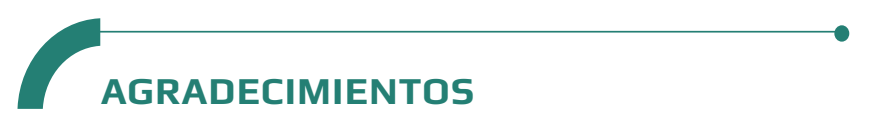

Agradecemos a los Ayuntamientos de la Ciudad de Mérida (2012-2015 y 2015-2018) las facilidades y recursos brindados para la realización de este proyecto. De igual manera, agradecemos a LN Rosemary Ávila, LN Mónica Barbosa, LN Alejandra Bolio, PLN Isabel Rodríguez, PLN Jimena Carrillo, $\mathrm{Br}$. Víctor Canto, Br. Karla Novelo y Br. Olivia Manzano su valiosa colaboración para el desarrollo de este trabajo.

\section{$\longrightarrow$ \\ CONFLICTO DE INTERESES}

Los recursos financieros para la realización de este trabajo fueron proporcionados por la Dirección de Desarrollo Integral Familiar Municipal de los Ayuntamientos de la Ciudad de Mérida, Yucatán, México (2012-2015 y 2015-2018). Las fuentes de financiación no han tenido participación en el 
diseño del estudio, la colección de datos, el análisis o la interpretación de estos, en la redacción del manuscrito o en la decisión de enviarlo para su publicación. Los autores expresan que no hay conflictos de intereses al redactar el manuscrito.

\section{REFERENCIAS}

(1) Martorell R, Melgar P, Maluccio JA, Stein AD, Rivera JA. The nutrition intervention improved adult human capital and economic productivity. J Nutr. 2010; 140(2): 411-4.

(2) Black RE, Allen LH, Bhutta ZA, Caulfield LE, de Onis M, Ezzati $M$, et al. Maternal and child undernutrition: global and regional exposures and health consequences. Lancet. 2008; 371(9608): 243-60.

(3) Gutiérrez JP, Rivera-Dommarco J, Shamah-Levy T, VillalpandoHernández S, Franco A, Cuevas-Nasu L, et al. Encuesta Nacional de Salud y Nutrición 2012. Resultados Nacionales. Cuernavaca, México: Instituto Nacional de Salud Pública (MX); 2012.

(4) Instituto Nacional de Salud Pública. Encuesta Nacional de Salud y Nutrición 2012. Resultados por entidad federativa, Yucatán. Cuernavaca, México: Instituto Nacional de Salud Pública (MX); 2013.

(5) Pereira A, Guedes AD, Verreschi ITN, Santos RD, Martinez TLR. Obesity and its association with other cardiovascular risk factors in school children in Itapetininga, Brazil. Arq Bras Cardiol. 2009; 93(3): 248-55.

(6) Rtveladze K, Marsh T, Barquera S, Sanchez Romero LM, Levy $D$, Melendez $G$, et al. Obesity prevalence in Mexico: impact on health and economic burden. Public Health Nutr. 2014; 17(1): 233-9.

(7) Secretaría de Salud (MX). Norma Oficial Mexicana NOM030-SSA2-2009, Para la prevención, detección, diagnóstico, tratamiento y control de la hipertensión arterial sistémica. NOM-030-SSA2-2009 2009.

(8) Secretaría de Salud (MX). Norma Oficial Mexicana NOM-015SSA2-2010, Para la prevención, tratamiento y control de la diabetes mellitus. NOM-015-SSA2-2010 2010.

(9) WHO Multicentre Growth Reference Study Group. WHO child growth standards: length/height-for-age, weight-for-age, weight-for-length, weight-for-height and body mass indexfor-age: methods and development. France: World Health Organization; 2006.

(10) Organización Mundial de la Salud. Patrones de Crecimiento del Niño de la OMS: Curso de Capacitación sobre evaluación del crecimiento del niño. Washington, DC, USA: Organización Mundial de la Salud; 2008.

(11) World Health Organization. Obesity: preventing and managing the global epidemic. Report of a WHO consultation. World Health Organ Tech Rep Ser. 2000; 894:i-xii, 1-253.

(12) Arroyo P, Fernández V, Loría A, Pardío J, Laviada H, VargasAncona $L$, et al. Obesidad, morfología corporal y presión arterial en grupos urbanos y rurales de Yucatán. Salud Pública Méx. 2007; 49(4): 274-85.

(13) Bastarrachea-Sosa R, Laviada-Molina H, Vargas-Ancona L. La obesidad y enfermedades relacionadas con la nutrición en Yucatán. Rev Endocrinol Nutr. 2001; 9(2): 73-6.

(14) Castro-Sansores C], Hernández-Escalante V, Arjona-Villicaña R. Prevalencia de Síndrome Metabólico en sujetos adultos que viven en Mérida, Yucatán, México. Rev Biomed. 2011; 22(2): 49-58.

(15) Aspectos teóricos básicos. En: Manual de procedimientos estandarizados para la vigilancia epidemiológica de la Diabetes Mellitus Tipo 2. México, D.F.: Dirección General de Epidemiología; 2012. p. 16-20.

(16) Rosado CA, Álvarez J, González A. Influencia de la herencia maya sobre el riesgo de diabetes mellitus tipo 2. Rev Endocrinol Nutr. 2001; 9(3): 122-5.

(17) Larrosa-Haro A, González-Pérez G], Vásquez-Garibay EM, Romero-Velarde E, Chávez-Palencia C, Salazar-Preciado LL, et al. Crecimiento acelerado en escolares obesos frente a escolares con peso saludable. Rev Med Inst Mex Seguro Soc. 2014; 52(Supl 1): S12-7.

(18) Kain J, Corvalán C, Lera L, Galván M, Weisstaub G, Uauy R. Asociación entre el índice de masa corporal y la talla desde el nacimiento hasta los 5 años en preescolares chilenos. Rev Med Chile. 2011; 139(5): 606-12.

(19) Bosy-Westphal A, Plachta-Danielzik S, Dörhöfer R-P, Müller M]. Short stature and obesity: positive association in adults but inverse association in children and adolescents. $\mathrm{Br}$ ] Nutr. 2009; 102(3): 453-61.

(20) Alcocer-Aguilar J, Hernández-Escalante VM, Marín-Cárdenas $A D$, Sansores CC, Cabrera-Araujo Z. Ingestión baja de hierro y anemia en escolares con sobrepreso y obesidad. Ciencia Humanismo Salud. 2015; 2(1): 4-9.

(21) Torres K, Marín A, Hoil J, Kim E. Ingesta de macronutrimentos e indicadores antropométricos en niños de un año de edad. Ciencia Humanismo Salud. 2015; 2(2): 43-52.

(22) Mendez N, Barrera-Pérez M, Palma-Solís M, Dickinson F, UicabPool G, Castillo-Burguete MT, et al. "You are not fat, you are hermosa»: Mexican caregivers share their perceptions about their role supporting their morbidly obese children. Hisp Health Care Int. 2014; 12(4): 174-82.

(23) Peralta-Romero J de J, Gómez-Zamudio JH, Estrada-Velasco B, Karam-Araujo R, Cruz-Lópeza M. Genética de la obesidad infantil. Rev Med Inst Mex Seguro Soc. 2014; 52(S1): 78-87.

(24) Valladares-Salgado A, Suárez-Sánchez F, Burguete-García Al, Cruza $M$. Epigenética de la obesidad infantil y de la diabetes. Rev Med Inst Mex Seguro Soc. 2014; 52(Supl 1): S88-93.

(25) Hurtado-López EF, Macías-Rosales R. Enfoque de la obesidad infantil desde la pediatría. Rev Med Inst Mex Seguro Soc. 2014; 52(Supl 1): S116-9. 